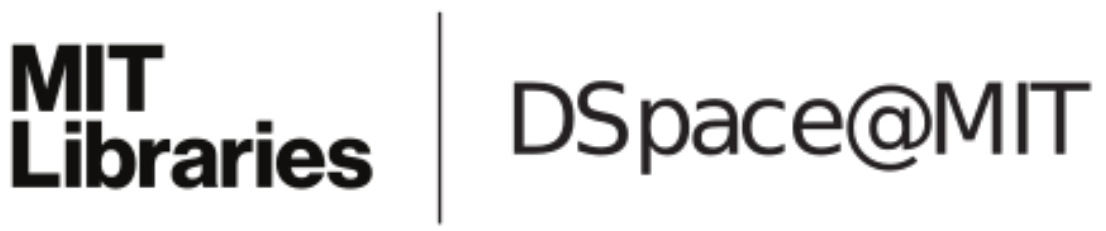

\author{
MIT Open Access Articles \\ Optimizing deep brain stimulation settings \\ using wearable sensing technology
}

The MIT Faculty has made this article openly available. Please share how this access benefits you. Your story matters.

Citation: Patel, S. et al. "Optimizing deep brain stimulation settings using wearable sensing technology." Neural Engineering, 2009. NER '09. 4th International IEEE/EMBS Conference on. 2009. 6-9. (C) 2009 IEEE

As Published: http://dx.doi.org/10.1109/NER.2009.5109221

Publisher: Institute of Electrical and Electronics Engineers

Persistent URL: http://hdl.handle.net/1721.1/58816

Version: Final published version: final published article, as it appeared in a journal, conference proceedings, or other formally published context

Terms of Use: Article is made available in accordance with the publisher's policy and may be subject to US copyright law. Please refer to the publisher's site for terms of use. 


\section{Optimizing Deep Brain Stimulation Settings Using Wearable Sensing Technology}

\author{
Shyamal Patel \\ Chiara Mancinelli \\ Richard Hughes \\ Department of PM\&R \\ Harvard Medical School \\ Boston, USA \\ spate119@partners.org, \\ cmancinelli@partners.org, \\ rhughes1@partners.org
}

\author{
Anthony Dalton \\ Bioelectronics Research Cluster \\ National University of Ireland \\ Galway, Ireland \\ afdalton@partners.org
}

\author{
Ludy Shih \\ Department of Neurology \\ Harvard Medical School, \\ Beth Israel Deaconess \\ Medical Center \\ Boston, USA \\ 1shih@caregroup.harvard.edu
}

\author{
Paolo Bonato \\ Department of PM\&R \\ Harvard Medical School \\ The Harvard-MIT \\ Division of Health \\ Sciences and \\ Technology \\ Boston, USA \\ pbonato@partners.org
}

\begin{abstract}
Parkinson's disease is a neurodegenerative movement disorder resulting in rigidity, bradykinesia (slowness), tremor and gait disorder. Deep brain stimulation (DBS) of the subthalamic nucleus has been shown to be effective in managing symptoms, but quantitative methods to facilitate the adjustment of the stimulator settings are needed. In this paper, we present preliminary results from a study aimed at investigating the use of wearable sensors to quantitatively track changes in the severity of symptoms in patients with Parkinson's disease undergoing programming of the stimulator. We developed a technique that relies upon features derived from wearable sensors to track changes in the severity of symptoms over a period during which patient's motor activities are monitored. Preliminary results indicate that wearable sensors could be utilized to help clinicians achieve optimal settings of the stimulator by providing quantitative feedback concerning the impact of different settings on the severity of Parkinsonian symptoms.
\end{abstract}

Keywords- Parkinson's Disease; Wearable Technology; Deep Brain Stimulation

\section{INTRODUCTION}

Parkinson's disease (PD) is the second most common neurodegenerative disease, affecting about $3 \%$ of the population over the age of 65 years and more than 500,000 US residents. The characteristic motor features of PD are development of rest tremor, bradykinesia, rigidity, and impairment of postural balance. The primary biochemical abnormality in PD is deficiency of dopamine due to the degeneration of neurons in the substantia nigra pars compacta. As a result, abnormal patterns of neural activity are present within the basal ganglia, including overactivation of the subthalamic nucleus, which contributes to development of tremor and other typical motor features.

Current therapy of PD is based on augmentation or replacement of dopamine, using levodopa or other drugs, which activate dopamine receptors. These therapies are often successful for some time, but in late stage PD most patients develop motor complications such as wearing off and dyskinesias. STN DBS is an effective way to manage late stage PD motor complications that develop over time in this patient population.

While STN DBS has shown dramatic results both in the short and the long term, several hypotheses have been put forth as possible causes of symptom reduction, but none of them is universally accepted. Although some attempts to systematically explore the DBS settings have been pursued [1][2], postoperative management in patients undergoing DBS for treating PD does not rely on methods that are grounded on proven models of the underlying mechanisms. The complex process of adjusting the neurostimulator parameters is rather driven by empirical observations that do not guarantee an optimal choice of the neurostimulator setting.

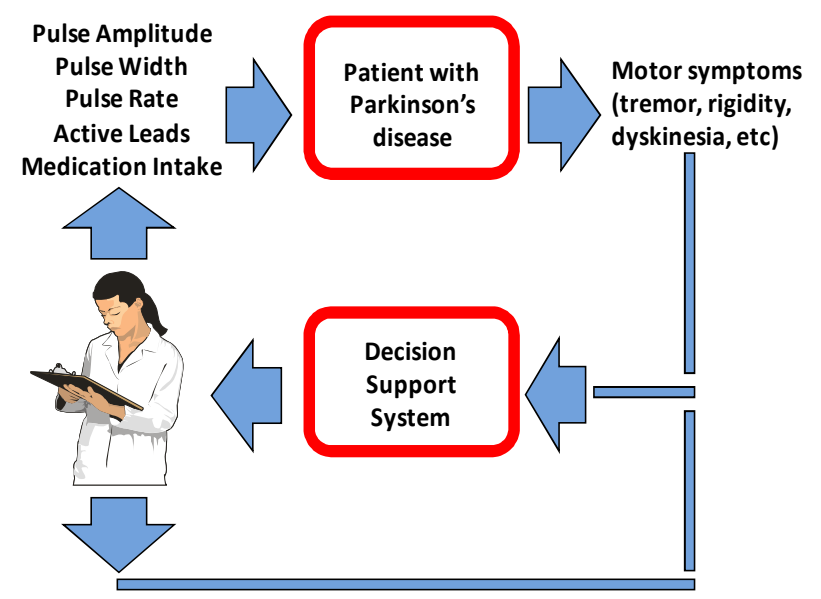

Figure 1 Schematic representation of the decision support system we envision to choose optimal DBS settings.

In earlier work by Ushe et al [3], the authors reported on the use of a single accelerometer to monitor tremor suppression in patients with essential tremor with changing DBS parameters. Our research team [4] used wearable sensors to monitor motor fluctuations in PD patients undergoing medication treatment. In a pilot study [5] on 5 patients, we tracked changes in the severity of symptoms as the DBS was turned from ON to OFF and back ON. In this 
paper, we present preliminary results from a new study in which we used wearable sensors to record data from patients undergoing DBS programming. The long-term objective of this study is to develop a decision support system (Figure 1) to provide feedback to clinicians regarding the efficacy of the stimulator settings thus reducing the time and effort required to achieve optimal outcomes.

\section{DBS PROGRAMMING}

DBS systems consist of a quadripolar electrode with an inter-contact distance of 1.5 or $0.5 \mathrm{~mm}$, an extension cable and an internal pulse generator (IPG) either controlling one (SOLETRA $^{\mathrm{TM}}$, Medtronic) or two KINETRA $^{\mathrm{TM}}$, Medtronic) DBS electrodes. Current is delivered through cylindrical electrode contacts of $1.27 \mathrm{~mm}$ diameter and $1.5 \mathrm{~mm}$ length. The relevant stimulation parameters, which can be controlled by means of an external console after implantation of the IPG, are electrode polarity and amplitude, duration, and frequency of the pulses. Each electrode contact can be programmed as anode or cathode in bipolar settings or as cathode for monopolar stimulation. Bipolar stimulation creates a more focused current field thus providing specificity. However, physicians often favor monopolar stimulation because it typically requires lower stimulation intensity than bipolar stimulation to achieve the same clinical benefit. Therapeutic amplitudes for DBS normally range between 1 and $3.5 \mathrm{~V}$. The commercial IPGs by Medtronic allow one to vary voltage in $0.1 \mathrm{~V}$ steps between 0 and $10.5 \mathrm{~V}$. However, current consumption of the neurostimulators by Medtronic is linear up to $3.6 \mathrm{~V}$ and rises abruptly above this value because a voltage doubler circuit is activated. The increase in amplitude raises significantly the current requirement and shortens battery life. Pulse width can be varied in steps between 60 and $450 \mu$ s however the typical values are between 60 and $90 \mu \mathrm{s}$. The pulse frequency can be set between 2 and $185 \mathrm{~Hz}$. However, the beneficial effects of stimulation are only observed at high frequencies, i.e. above $100 \mathrm{~Hz}$.

These general criteria for choosing the parameters of the neurostimulator result in a "decision space" defined by pulse amplitude ranging between 1 and $3.5 \mathrm{~V}$, pulse width between 60 and $90 \mu \mathrm{s}$, frequency between 110 and $150 \mathrm{~Hz}$, and a choice of monopolar or bipolar stimulation across the quadripolar electrode. To make this decision space more difficult to explore, responses related to different target symptoms are associated with different time constants. For instance, while tremor and rigidity respond within seconds, the effect of stimulation on bradykinesia and dystonia evolves over hours to days, and dyskinesia may show changes over weeks. Furthermore, a complex dynamic behavior marks postoperative management of medications. Levodopa therapy and dopamine agonist treatment need to be adjusted after implant of the neurostimulator. Cyclical variations of the response to STN stimulation are observed with medication intake. Fluctuations of the target symptoms are also observed over days and weeks. To accomplish the goals of adjusting the stimulator parameters, namely 1) to maximize symptom suppression, 2) to minimize side effects, and 3) to maximize neurostimulator battery life, becomes therefore a very challenging task.

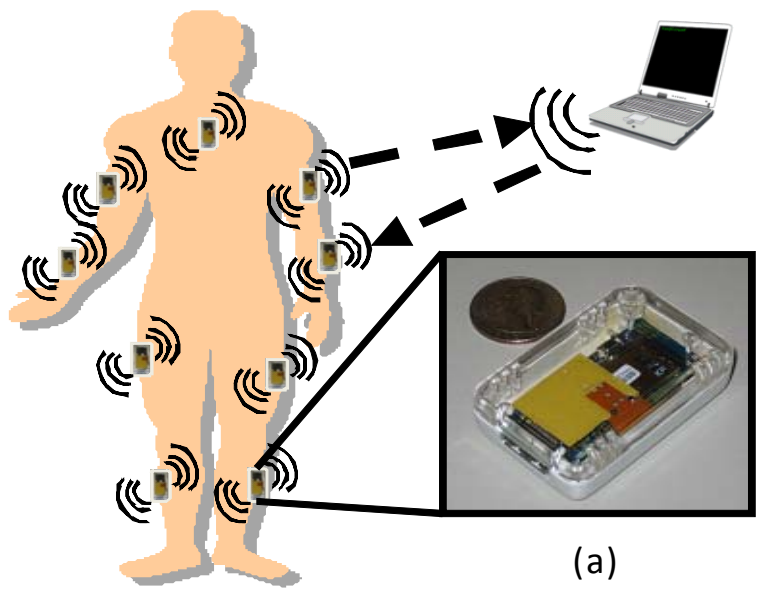

Figure 2 Sensor setup utilized for the data collection and (a) SHIMMER wireless sensor unit.

\section{METHODS}

\section{A. Data Collection}

We used the SHIMMER wireless system by Intel ${ }^{\circledR}$ (Figure 2) for data collection. This platform consists of a TI MSP430 microprocessor; a Chipcon CC2420 IEEE 802.15.4 2.4 GHz radio; a MicroSD card slot; and a tri-axial MEMS accelerometer (Freescale MMA7260Q). Compared with other wireless sensors, SHIMMER achieves a smaller footprint using conventional board technology and integrates a lithium-polymer battery.

So fat, in the ongoing study herein described, we have collected data from 4 patients. Tri-axial accelerometer data was recorded at $100 \mathrm{~Hz}$ while subjects performed standardized motor tasks from the Unified Parkinson's Disease Rating Scale (UPDRS). Each motor task was performed for about $30 \mathrm{~s}$ or for a fixed number of repetitions. Each subject's movements were recorded using 9 accelerometers. The accelerometers were placed bilaterally at the midpoint of the forearm and the upper arm, on the shank approximately $10 \mathrm{~cm}$ above the ankle, on the thigh approximately $10 \mathrm{~cm}$ above the knee and on the upper back (see Figure 2).

Figure 3 shows schematically the sequence of the testing sessions. Video recordings of each session were gathered for later review and UPDRS scoring. Data collection was performed before, during and after DBS programming on the day of clinical adjustment of DBS settings. During the clinical visit, data was collected before any adjustment was made, after testing each electrode contact, and at the end of the adjustment session before the patient was ready to leave the clinic. Also, two weeks after each session of DBS adjustments we gathered data for 3-4 hrs over 7 testing 
session. Each session was about 5-10 min long. Between each two testing sessions, patients were allowed to rest for about 20-25 min. During the course of the study, data was collected during 4 DBS adjustment visits and 3 times in between DBS programming visits.

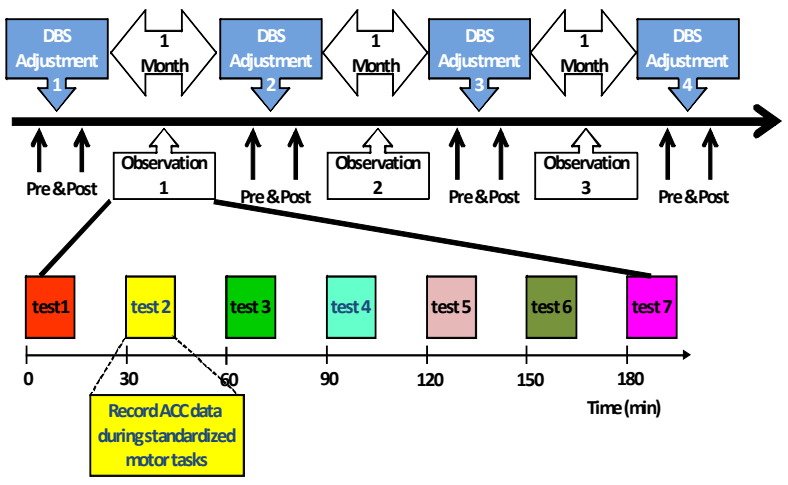

Figure 3 Schematic representation of the data collection procedure.

\section{B. Data Analysis}

Before extracting features, the raw accelerometer data was high-pass filtered with a cutoff frequency of $1 \mathrm{~Hz}$ to attenuate components associated with gross changes in the orientation of body segments. Also the data was low-pass filtered with a cutoff frequency of $15 \mathrm{~Hz}$ to remove the high frequency noise. The data from the $\mathrm{x}-, \mathrm{y}$ - and $\mathrm{z}$-axis of each accelerometer was combined by taking the square root of the sum of squares of each axis.

Sets of 30 epochs of $4 \mathrm{~s}$ were randomly selected from the sensor data recorded during performance of each motor task, for each trial. We chose features that represent characteristics of movements such as intensity, modulation, rate, periodicity, and coordination of movement. Intensity was measured as the root-mean-square (RMS) value of the detrended accelerometer signal. The modulation of the output of each sensor was used to represent dynamic characteristics of the tasks, and was calculated as the range of the auto-covariance function of each channel. Large values of this feature were indicative of intervals of rapid movements interspersed with intervals of slow movements. Rate of movement was represented by the dominant frequency component below $10 \mathrm{~Hz}$. Periodicity was measured by computing the ratio of the energy of the dominant frequency component to the total energy below $10 \mathrm{~Hz}$. Coordination between body segments on the left and right side and proximal and distal segments was captured in three aspects: magnitude (obtained by calculating the correlation coefficient), delay (estimated as the time lag corresponding to the peak of the cross-correlation function) and similarity (measured by the value of the peak of the cross-correlation function). An estimate of entropy of the signal [7] was used as a measure of signal complexity.

The results presented in this abstract are based on visual inspection of the data. First we correlated the changes in clinical scores of severity of symptoms with changes in feature value. Secondly we inspected the feature space using the Sammons mapping technique. Sammons mapping [6] is a nonlinear transformation technique that reduces data dimensionality by preserving the distance between points in lower dimensions. It is a useful method for achieving visual examination of the data structure. We utilized this technique to derive two-dimensional projections of the feature sets.

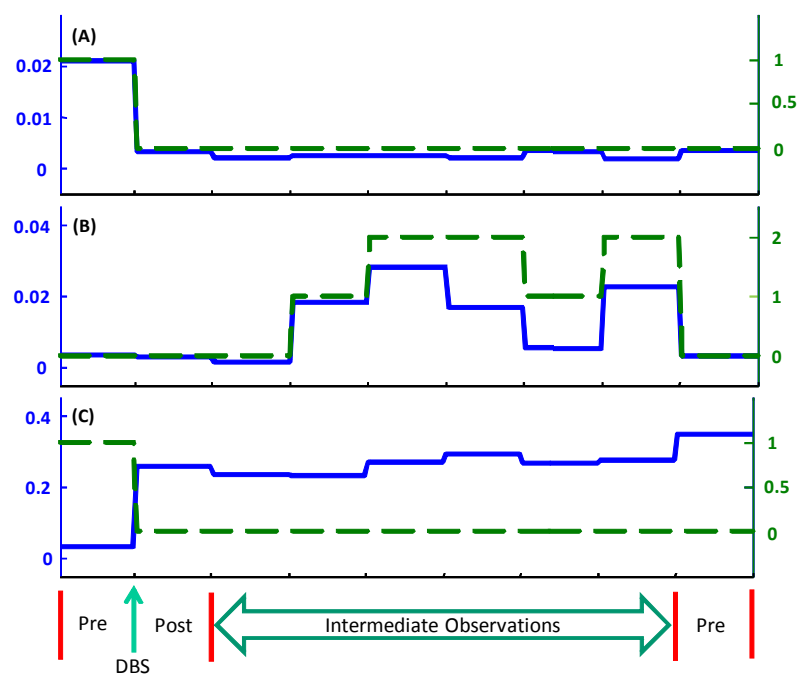

Figure 4 Plot of the RMS value, extracted from the right forearm, relative to the clinical score for (a) tremor (data recorded during steady holding of arms out front while seated), (b) dyskinesia (data recorded during the finger to nose task with left hand), and (c) bradykinesia (data recorded during finger to nose with right hand). RMS value is shown by the bold blue line and clinical scores are shown by the dashed green line.

\section{RESULTS}

Analysis of the feature space shows that distinct patterns of feature values are associated with changes in the severity of Parkinsonian symptoms. In the following, we provide a detailed description of the results derived from the analysis of data from one patient. Similar results were obtained from other patients. Figure 4 shows changes in clinical scores for tremor, bradykinesia and dyskinesia over a period of several days. It also shows measures of the RMS value derived from the accelerometer time series recorded from the right forearm during the same sessions from which the clinical scores were derived. During the first session, we performed DBS adjustments. Data was gathered before and after adjusting the DBS settings. During the second session, we recorded multiple trials during a period of several hours to capture changes in the severity of symptoms that mark motor fluctuations. The second session was scheduled about 2 weeks following the first DBS programming visit. The third session (the last for which results are shown in Figure 4) corresponded to the second DBS programming visit and was scheduled about one month after the first visit. Data is shown for the recordings performed before (pre-DBS) and after (post-DBS) the actual DBS programming during the first session, for the 7 trials performed during the second 
session, and for the recordings performed before (pre-DBS) the DBS programming visit during the third session.

Figure 4 suggests that changes in RMS value are highly correlated with changes in the clinical scores for all three symptoms we monitored during the experiments (i.e. tremor, bradykinesia and dyskinesia). Tremor and dyskinesia are symptoms related to involuntary movement; hence, as the clinical score goes down the intensity of movement (represented by the RMS value in figure 4(a) \& 4(b)) goes down as well. Bradykinesia represents the rigidity or slowness of movement; hence, as the clinical score goes down the ability of a patient to perform a task improves (indicated by the increased RMS value in figure 4(c)). Also, it appears that the severity of tremor and bradykinesia changed significantly in response to the adjustment in DBS settings performed during the first session.

It is worth emphasizing that, when patients undergo the first DBS programming visit, they are instructed to withdraw their medications for about 12 hours prior to the visit (this procedure is referred to as "practically-defined off"). Consequently, it is expected that patients show virtually no dyskinesia during the first session and therefore seeking correlation between dyskinesia scores and RMS values for this session is practically meaningless. During the second session, patients are observed during a period of normal medication intake. Therefore, a correlation between the clinical score for dyskinesia and the RMS values can be meaningfully sought. Figure 4 shows that clinical scores for dyskinesia and RMS values are highly correlated during the second recording session. Such correlation is maintained when data from all the sessions (including the pre-DBS observations during the third session) is considered. Although a visual correlation cannot be used to draw conclusions, it indicates the ability of features derived from accelerometer data to capture changes in severity of symptoms over a period of several days.

A Sammons' map for the data collected over the three visits is shown in Figure 5. The points in Figure 5 are labeled by clinical score for bradykinesia. By visual inspection, it is apparent that there is a good separation between clusters associated with different clinical scores. These results suggest that information extracted from accelerometer data can be used to build models that can provide an accurate prediction of clinical score values.

\section{DISCUSSION AND FUTURE WORK}

The results obtained so far provide preliminary evidence of the potential of wearable sensors for facilitating the process of seeking optimal stimulator settings in patients with Parkinson's disease undergoing DBS. The observations performed over multiple visits allowed us to explore the hypothesis that features extracted from wearable sensor data change in a way that correlates with clinical scores. Our results suggest that the hypothesis we formulated holds, thus indicating that wearable sensors could be used to track changes in Parkinsonian symptoms over long periods therefore providing clinical feedback concerning the effect of different DBS settings. If future clinical studies confirm our preliminary results, we could envision utilizing a wearable system during the DBS programming period. Information gathered with such system could be of great help during the initial phases of the DBS programming period as clinicians could be provided with reliable quantitative information concerning the impact of exploratory adjustments in DBS settings. Availability of this information would be expected to facilitate the process aimed to achieve optimum outcomes.

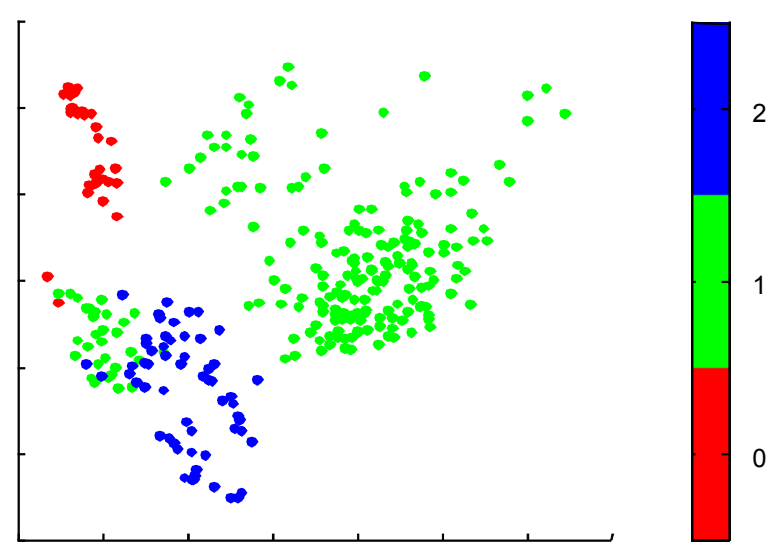

Figure 5 Sammons mapping projection of the feature set. The patient is performing pronation-supination movements of the hand. The points are labeled by clinical score for bradykinesia.

\section{REFERENCES}

[1] J. Volkmann, J. Herzog, F. Kopper, G. Deuschl, "Introduction to the Programming of Deep Brain Stimulators", Movement Disorders, vol. 17, suppl. 3, p. S181-S187, 2002.

[2] R. Kumar, "Methods for Programming and Patient Management with Deep Brain Stimulation of the Globus Pallidus for the Treatment of Advanced Parkinson's Disease and Dystonia", Movement Disorders, vol. 17, suppl. 3, p. S198-S207, 2002.

[3] M. Ushe, J.W. Mink, F.J. Revilla, A. Wernle, G.P. Schneider, L. McGee-Minnich, M. Hong, K.M. Rich, K.E. Lyons, R. Pahwa, J.S. Perlmutter, "Effect of Stimulation Frequency on Tremor Suppression in Essential Tremor", Movement Disorders, vol. 19, n. 10, p. 1163-1168, 2004.

[4] S. Patel, D. Sherrill, R. Hughes, T. Hester, N. Huggins, T. LieNemeth, D. Standaert, P. Bonato, "Analysis of the Severity of Dyskinesia in Patients with Parkinson's Disease via Wearable Sensors", BSN2006 - International Workshop on Wearable and Implantable Body Sensor Networks, Boston MA, April 3-5, 2006.

[5] S. Patel, T. Hester, R. Hughes, N. Huggins, A. Flaherty, D. Standaert, J. Growdon, P. Bonato, "Processing Wearable Sensor Data to Optimize Deep-Brain Stimulation", IEEE Pervasive Computing, vol. 7, n. 1, p. 56-61, Jan.-Mar. 2008.

[6] J.W. Sammon, "A Nonlinear Mapping for Data Structure Analysis", IEEE Transactions on Computers, vol. C-18, n. 5, p. 401-409, May 1969.

[7] R. Moddemeijer, "On Estimation of Entropy and Mutual Information of Continuous Distributions", Signal Processing, 1989, vol. 16, n. 3, p. $233-246$

This work was supported by the Center for Integration of Medicine and Innovative Technology (CIMIT) under the project entitled "Enhancing Parkinson's Control Therapy by Stimulation of the Subthalamic Nucleus via Measures of Motor Response". 